\title{
Evaluación de la solidez del color en tejidos de lana y alpaca tinturados con biocolorantes extraídos de plantas y animales
}

\author{
Evaluation of color fastness in wool and alpaca fabric dyed \\ with biocolorants extracted from plants and animals
}

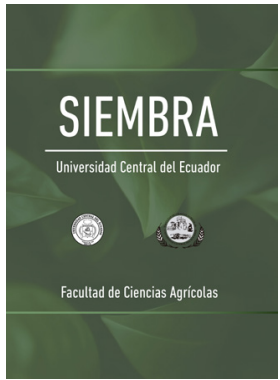

\author{
Cecilia Palacios-Ochoa ${ }^{1 *}$, María Elisa Guillén Serrano ${ }^{2}$, \\ David Christopher Siddons ${ }^{3}$
}

\footnotetext{
${ }^{11}$ Universidad del Azuay, Facultad de Diseño Arquitectura y Arte, Carrera de Diseño Textil e Indumentaria. Apdo. 982. Cuenca, Ecuador.

凶cpalacios@uazuay.edu.ec D. https://orcid.org/0000-0003-0167-4771

${ }^{2}$ Universidad del Azuay, Facultad de Diseño Arquitectura y Arte, Carrera de Diseño Textil e Indumentaria. Apdo. 982. Cuenca, Ecuador. Ecuador.

$\bowtie$ eguillen@uazuay.edu.ec

B. https://orcid.org/0000-0003-0837-0383

${ }^{3}$ Universidad del Azuay, Facultad de Ciencia y Tecnología, Carrera Biología Ecología y Gestión. Apdo. 982. Cuenca, Ecuador. Ecuador.

凶dsiddons@uazuay.edu.ec

Q https://orcid.org/0000-0003-3305-2969
}

*Autor de correspondencia: cpalacios@uazuay.edu.ec

\section{SIEMBRA}

https://revistadigital.uce.edu.ec/index.php/SIEMBRA ISSN-e: $2477-8850$

\section{ISSN: $1390-8928$}

Periodicidad: semestral

vol. 8, núm. 2, 2021

siembra.fag@uce.edu.ec

DOI: https://doi.org/10.29166/siembra.v8i2.2917

\section{Resumen}

Esta investigación consistió en evaluar la solidez del color de tejidos de lana y alpaca tinturados con colorantes extraídos de plantas nativas de la provincia del Azuay en el sur ecuatoriano. Las plantas tintóreas que se utilizaron fueron: Lomatia hirsuta (garau), Baccharis latifolia (chilca), Juglans neotropica (nogal), Brachyotum confertum (killuyuyo) y además se empleó el colorante de origen animal Dactilopius coccus (cochinilla). Se utilizó la metodología ancestral para el teñido de los hilos, con los que se prepararon muestras de tejido de punto que sirvieron para realizar las pruebas de solidez del color a la luz artificial, lavado manual con jabón neutro y alcalino, y la prueba de frote en húmedo y en seco. La evaluación de la pérdida de color de los tejidos se realizó mediante un colorímetro, se midió el color antes y después de las pruebas y se calculó la diferencia total de color $(\Delta \mathrm{E})$. Se encontró una mayor variación del color en el tratamiento de lavado de las muestras con jabón alcalino (el valor más alto fue 32) y en el resto de las pruebas se observó una mínima diferencia de color (1,4 en promedio). Haciendo la relación con la escala de grises los resultados corresponden a la calificación de satisfactorio para la mayor parte de muestras, por lo que se demostró que el color de los tejidos al utilizar garau, chilca, nogal, killuyuyo y cochinilla, es estable a la mayoría de los factores ensayados. Estos resultados son importantes porque sirven de base para la aplicación de algunos procesos para obtener una mayor resistencia del color en indumentaria elaborada con hilos teñidos con biocolorantes.

Palabras clave: colorantes, colorantes vegetales, colorantes animales, resistencia, pruebas.

\begin{abstract}
This research evaluated the color fastness of natural dyes extracted from native plants and animals in the province of Azuay, southern Ecuador, on knitted fabrics made from wool and alpaca yarns. Native plants dye was made from: Lomatia hirsute (garau), Baccharis latifolia (chilca), Junglas neotrópica (nogal) Brachyotum confertum (killuyuyo) while an animal-based dye was also extracted from Dactilopius coccus (cochinilla). Traditional ancestral methods of dyeing the yarn
\end{abstract}


were used. Samples of knitted fabric were prepared to perform the tests of color fastness with artificial light, manual washing with neutral and alkaline soap, and a wet and dry rub test. The evaluation of the color loss of the fabrics was made by means of a colorimeter, the color was measured before and after the tests and the total color difference $(\Delta \mathrm{E})$ was calculated. A greater color variation was found in the washing treatment of the samples with alkaline soap (highest value $=32$ ) and a minimum color difference in the other tests (mean
$=1.4$ ). In correlation with the grayscale, these results suggest an ideal rating. Which demonstrates that the color of the fabrics when using garau, chilca, nogal, killuyuyo and cochinilla is stable to most of factors tested. These results are important because they serve as a baseline for the application of further processes to obtain greater color resistance in clothing made with yarn dyed with biocolorants.

Keywords: dyeing, plant-dyes, animal-dyes, resistance, tests.

\section{Introducción}

En la actualidad, el uso de colorantes naturales ha tenido un desarrollo notable en algunos sectores de la población, esto se debe a la necesidad de contribuir a la protección del ambiente, y de optar por productos menos dañinos para la salud. Estos colorantes se denominan también eco-tintes por ser respetuosos con la naturaleza y se los obtiene de varias fuentes naturales entre las que se cuentan los vegetales, animales y microorganismos (Vigueras y Portillo, 2001).

En lo que respecta a la historia de los colorantes naturales en el Ecuador, no se conoce la fecha exacta en la que se empezaron a utilizar, pero se tiene algunos datos de la época de la Colonia cuando alcanzaron una considerable importancia y se llevaba cochinilla al viejo continente como una mercancía muy valiosa (Jaramillo, 1988). Los indígenas los usaban en un proceso tintóreo, cuyo conocimiento fue pasando de generación en generación hasta que en nuestros días es algo casi olvidado (Guirola, 2010). Sin embargo, estas técnicas merecen ser rescatadas por ser parte del patrimonio cultural inmaterial (Palacios-Ochoa y Ullauri, 2020). Las plantas tintóreas comúnmente empleadas hasta nuestros días para la extracción de los colorantes son: nogal, chilca, molle, retama, mora de castilla, así como también la cochinilla extraída del insecto Dactilopius coccus (Jaramillo, 1988; Palacios-Ochoa y Ullauri, 2020). Estos colorantes son afines a las fibras de origen natural, presentando una mayor compatibilidad con las de naturaleza proteica como la lana de oveja, fibra de alpaca y seda. Menor afinidad tienen las fibras de origen vegetal como el algodón, el bambú, etc. (Alonso, 2015).

Para que las técnicas ancestrales de tinturado sean valoradas es importante emprender estudios que sirvan para demostrar los usos que se pueden dar a las fibras tinturadas con colorantes naturales. Los textiles tejidos representan una alternativa que permite apreciar las bondades de los colores que nos ofrece la naturaleza, y al mismo tiempo sirven para motivar a diseñadores y artesanos al uso de las técnicas de tinturado natural en la innovación de productos textiles con características de exclusividad y con la impronta de lo natural. Algunas técnicas de tejido son el tejido plano, cuando se usan los telares; el tejido de punto que es un tejido que se realiza mediante el entrelazamiento de bucles utilizando dos agujas o múltiples agujas. Para el denominado tejido de punto por urdimbre que es el que se utiliza comúnmente para elaboración de estructuras textiles a mano, la aguja es alimentada por un hilo en el sentido de las columnas formando una malla (Alonso, 2015). Los tejidos de fibras naturales que se destinan al diseño de prendas resultan con un atractivo adicional cuando son tinturados con tintes extraídos de recursos naturales (Martínez, 2011).

Por otro lado, la aplicación de biocolorantes en la elaboración de textiles requiere la realización de análisis de la resistencia del color ya sea en los hilos o tejidos. Se debe probar la resistencia a diferentes factores ambientales o los derivados del uso mismo de los textiles, esto incluye una serie de ensayos sobre los tejidos para comprobar la eficacia que aportan al producto final (Carrera-Gallissà, 2015).

En el caso de textiles teñidos es importante determinar la solidez o resistencia del color del textil cuando es expuesto a la luz, al lavado y a un roce de su superficie, acciones frente a las que el tinte debería ser firme para ostentar una buena calidad (Alonso, 2015). Por lo que el objetivo del presente trabajo fue analizar la resistencia del color de muestras de tejido de punto elaboradas con fibra de oveja y alpaca, tinturadas con colorantes extraídos de algunas plantas nativas de la provincia del Azuay en el sur de Ecuador. Se utilizaron plantas nativas como Lomatia hirsuta (garau), Baccharis latifolia (chilca), Juglans neotrópica (nogal), Brachyotum confertum (killuyuyo) y además se empleó el colorante de origen animal cochinilla, obtenida del insecto Dactilopius coccus. 
El uso de plantas nativas para la experimentación tiene gran importancia porque son los recursos que utilizan en la actualidad los artesanos para el tinturado, se las obtiene con facilidad en los lugares donde ellos habitan. La cochinilla es un colorante que también lo usan, pero ésta la compran de localidades cercanas o del vecino país Perú. Los conocimientos obtenidos de esta investigación pueden ser retribuidos a las comunidades que se dedican al tinturado natural lo que servirá para mejorar la calidad de los productos artesanales y dinamizar su economía.

Con la evaluación de la resistencia del tinturado se pudo determinar la calidad de los tejidos elaborados con las fibras de lana de oveja y alpaca tinturadas con los colorantes extraídos del nogal, garau, chilca, killuyuyo y de la cochinilla. Se llegó a definir que el tinturado es de buena calidad lo que es importante porque motiva a diseñadores y artesanos al uso de las metodologías ancestrales de tinturado para generar hilos y tejidos que les permitirá obtener productos atractivos, exclusivos y con un valor agregado por estar constituidos en su totalidad con recursos naturales.

\section{Materiales y métodos}

\subsection{Tinturado de los hilos}

Para teñir los hilos de lana y alpaca se aplicaron las técnicas ancestrales de tinturado, que fueron recuperadas de los artesanos y optimizadas a partir de una investigación previa (Palacios-Ochoa y Ullauri, 2020).

\subsubsection{Extracción del colorante}

Las plantas utilizadas para la extracción de los colorantes fueron: chilca, nogal, killuyuyo y garau y en el caso del colorante cochinilla éste se obtuvo en polvo listo para el uso. La cantidad de vegetal empleada fue de $200 \%$ en peso de la fibra y la proporción de cochinilla fue de $10 \%$ en peso de fibra a teñir. La extracción del colorante consistió en someter el material vegetal a ebullición en una cantidad de $1000 \mathrm{ml}$ de agua, por el lapso de 45 minutos y su posterior filtrado para eliminar los residuos. En el caso de la cochinilla el polvo se colocó en el agua y se sometió a ebullición por 5 minutos.

\subsubsection{Preparación del hilo}

Se inició con la preparación de madejas de hilo de $20 \mathrm{~g}$ cada una, el hilo utilizado fue de color original. Las madejas se colocaron en una solución de jabón neutro líquido al $0,5 \% \mathrm{v} / \mathrm{v}$, preparada en una cantidad de 500 $\mathrm{ml}$, que es suficiente para cubrir la madeja, se dejó por el lapso de 15 minutos. Con este tratamiento las fibras presentan una mayor superficie de contacto con el colorante.

\subsubsection{Mordentado}

Se realizó el mordentado directo, esto es, colocando el mordiente en el baño tintóreo, este tipo de mordentado es más rápido elimina una etapa en el proceso y no presenta diferencias con los resultados que se obtienen cuando se aplica un premordentado. Para esto se debe pesar el mordiente y disolver en $20 \mathrm{ml}$ de agua, se calienta si es necesario para que se disuelva completamente y se agrega al tinte.

Como mordientes se usaron el sulfato de hierro II y alumbre, ambos en la proporción del $10 \%$ con respecto al peso de la fibra, esto es $2 \mathrm{~g}$ de cada uno por madeja de hilo de $20 \mathrm{~g}$. Para el tono anaranjado con la cochinilla se usó además del alumbre, el ácido oxálico en proporción de $25 \%$ sobre el peso de la fibra.

\subsubsection{Tinturado}

El proceso de impregnación del tinte en las fibras se realizó colocando las madejas en la preparación formada por el tinte y el mordiente y sometiéndola a una temperatura de $80{ }^{\circ} \mathrm{C}$ por 30 minutos, para lo cual se utilizó un baño María de tinturado. La relación del baño de tinte fue de 50 a 1 con respecto al volumen de la fibra y se controló el pH de los baños, en el caso de la cochinilla el pH fue de 5,5, chilca 5, killuyuyo 5,5, garau 5 y nogal 4,5. Finalmente, se retiraron las madejas del baño y se lavaron con agua corriente, cuyo pH fue de 6,5. Con el lavado se eliminó el exceso de colorante y se dejaron secar a la sombra. 


\subsection{Elaboración de muestras de tejidos}

Los hilos de lana y alpaca teñidos con cada uno de los colorantes extraídos, se usaron para la elaboración de muestras o probetas de tejido para cada una de las pruebas de calidad, en las siguientes medidas: $20 \times 10 \mathrm{~cm}$ para la prueba de lavado, $10,5 \times 6 \mathrm{~cm}$ para la de solidez a la luz y $10 \times 4 \mathrm{~cm}$ para la prueba de frote. La puntada que se utilizó para los tejidos fue la denominada punto liso o jersey.

\subsection{Pruebas de solidez del color}

Para establecer la resistencia del color se realizaron algunos tratamientos como son: resistencia a la exposición a luz artificial, lavado manual con jabón neutro y con jabón alcalino y frote en húmedo y en seco.

\subsubsection{Prueba de solidez a la luz}

Esta prueba se realizó mediante la aplicación de luz artificial con el equipo Lumitester MBTL, el mismo que funciona con una lámpara de mercurio halogenado de electrodos de tungsteno y vapor de mercurio a $400 \mathrm{~W}$. La exposición a esta lámpara se correlaciona muy bien, tanto con el arco de xenón como con la luz del día, esta prueba se aplicó durante el tiempo de 3 horas y se utilizó una probeta de tejido de $6 \mathrm{~cm}$ x 10,5 cm. Se hizo la medición de color al inicio de la prueba y una vez terminado el tiempo de exposición con el colorímetro Precise Colorimeter CS-210, CHN Spec.

\subsubsection{Prueba de lavado manual}

Consistió en usar jabón neutro $(\mathrm{pH} 7)$ y alcalino $(\mathrm{pH} 8)$, las probetas que se utilizaron para esto fueron de $20 \mathrm{x}$ $10 \mathrm{~cm}$, se realizó el lavado manual con una solución de jabón neutro al $0,5 \% \mathrm{v} / \mathrm{v}$ y otra prueba con una solución de jabón en polvo alcalino $0,5 \% \mathrm{~m} / \mathrm{v}$, en agua corriente $(\mathrm{pH} 6,5)$ durante 30 minutos, simulando 5 lavados manuales como lo estipula la Norma ISO 105-C06:2010.

\subsubsection{Prueba de frote}

La prueba de resistencia al roce o de frote, se ejecutó con un frotímetro mecánico, AATCC CROCKMETER, se usó la probeta de tejido de $10 \times 4 \mathrm{~cm}$, que se coloca en la superficie inferior recubierta de lija que posee el aparato, sobre esta pasa un pequeño dispositivo cilíndrico móvil, que va recubierto con una tela testigo blanca sobre la que se obtiene un manchado por efecto del roce en caso de pérdida del color por parte de la muestra. El tejido testigo cumple la norma ISO 105-F-Parte F09 y es de 5 x $5 \mathrm{~cm}$ (Alonso, 2015). Se frotó por 20 veces la muestra humedecida en agua $(\mathrm{pH} 6,5)$ y de igual manera se procedió con la muestra seca. La tela testigo se comparó con la escala de grises para transferencia del color y también se midió el color de la probeta antes y después del tratamiento utilizando el colorímetro.

La solidez del color se evaluó mediante el colorímetro, midiendo el color de las probetas teñidas antes y después de los tratamientos de exposición a la luz, lavado y frote. Mediante el colorímetro se establecieron los valores de color y la variación total del color de las muestras o $\Delta \mathrm{E}$, así como la $\Delta \mathrm{L}^{*}$ (variación de la luminosidad), $\Delta \mathrm{a}^{*}$ (variación en el rango rojo y verde) y $\Delta \mathrm{b}^{*}$ (variación en el rango amarillo y azul) en la escala CIEL*a*b*. CIE es la Comisión Internacional de la Iluminación que tiene desarrollado el modelo cromático para describir los colores que puede percibir el ojo humano (Konica Minolta Sensing Americas, Inc., 2016). Además, se relacionó con la valoración que establece la escala de grises.

\section{Resultados y Discusión}

Los resultados de la diferencia total de color o $\Delta \mathrm{E}$, y los valores de la luminosidad antes y después de los tratamientos, así como las diferencias $\Delta \mathrm{L}^{*}, \Delta \mathrm{a}^{*} \mathrm{y} \Delta \mathrm{b}^{*}$ de los diferentes ensayos, se muestran en las Tablas 1 y 2 . Cada valor corresponde al promedio de la evaluación de más de tres puntos por medio del colorímetro. 
Tabla 1. Lecturas del colorímetro antes y después de las pruebas aplicadas a los tejidos de fibra de alpaca y valoración según la escala de grises Table 1. Colorimeter readings before and after the tests applied to the alpaca fiber fabrics and the evaluation according to the gray scale.

\begin{tabular}{|c|c|c|c|c|c|c|c|c|}
\hline Alpaca & Codificación* & $\mathbf{L a}$ & Ld & $\Delta \mathbf{E}$ & $\Delta \mathbf{L}^{*}$ & $\Delta a^{*}$ & $\Delta \mathbf{b}^{*}$ & $\begin{array}{l}\text { Escala } \\
\text { grises }\end{array}$ \\
\hline \multirow{5}{*}{ cochinilla rosa } & 02L & 53,26 & 53,71 & 1,25 & 0,45 & $-0,91$ & 0,74 & 4 \\
\hline & $02 \mathrm{~A}$ & 53,26 & 66,69 & 19,78 & 13,43 & $-13,92$ & 4,13 & 1 \\
\hline & $02 \mathrm{~N}$ & 53,26 & 53,71 & 1,25 & 0,45 & $-0,91$ & 0,74 & 4 \\
\hline & $02 \mathrm{H}$ & 53,26 & 53,71 & 1,25 & 0,45 & $-0,91$ & 0,74 & $3-4$ \\
\hline & $02 \mathrm{~S}$ & 53,26 & 53,71 & 1,25 & 0,45 & $-0,91$ & 0,74 & $3-4$ \\
\hline \multirow{5}{*}{$\begin{array}{l}\text { cochinilla } \\
\text { naranja }\end{array}$} & $03 \mathrm{~L}$ & 57,85 & 57,07 & 1,27 & $-0,78$ & 0,9 & 0,45 & $4-5$ \\
\hline & $03 \mathrm{~A}$ & 57,53 & 42,09 & 32,48 & $-15,44$ & 7,69 & $-27,52$ & 1 \\
\hline & $03 \mathrm{~N}$ & 57,53 & 56,66 & 2,65 & $-0,88$ & 1,24 & $-2,17$ & $3-4$ \\
\hline & $03 \mathrm{H}$ & 57,85 & 57,07 & 1,27 & $-0,78$ & 0,9 & 0,45 & $4-5$ \\
\hline & $03 \mathrm{~S}$ & 57,85 & 57,07 & 1,27 & $-0,78$ & 0,9 & 0,45 & $4-5$ \\
\hline \multirow{5}{*}{ nogal } & $03 \mathrm{~L}$ & 62,56 & 59,8 & 2,88 & $-2,76$ & 0,7 & 0,45 & $3-4$ \\
\hline & $03 \mathrm{~A}$ & 62,56 & 66,28 & 3,74 & 3,72 & $-0,02$ & $-0,35$ & 3 \\
\hline & $05 \mathrm{~N}$ & 62,56 & 64,28 & 1,93 & 1,72 & 0,44 & 0,76 & 4 \\
\hline & $05 \mathrm{H}$ & 62,56 & 64,28 & 1,93 & 1,72 & 0,44 & 0,76 & $4-5$ \\
\hline & $05 \mathrm{~S}$ & 62,56 & 64,28 & 1,93 & 1,72 & 0,44 & 0,76 & $4-5$ \\
\hline \multirow{5}{*}{ Garau } & $07 \mathrm{~L}$ & 62,31 & 62,04 & 0,65 & $-0,27$ & $-0,31$ & $-0,5$ & $4-5$ \\
\hline & 07A & 61,52 & 65,75 & 5,26 & 4,23 & 0,36 & 3,1 & $2-3$ \\
\hline & $07 \mathrm{~N}$ & 61,52 & 61,56 & 0,51 & 0,04 & $-0,03$ & 0,51 & $4-5$ \\
\hline & $07 \mathrm{H}$ & 61,52 & 61,56 & 0,51 & 0,04 & $-0,03$ & 0,51 & 4 \\
\hline & $07 \mathrm{~S}$ & 61,52 & 61,56 & 0,51 & 0,04 & $-0,03$ & 0,51 & 4 \\
\hline \multirow{5}{*}{ chilca } & 09L & 42,68 & 43,46 & 0,79 & 0,77 & 0,07 & 0,13 & $4-5$ \\
\hline & 09A & 42,68 & 41,59 & 4,11 & $-1,1$ & 3,05 & 2,53 & 3 \\
\hline & $09 \mathrm{~N}$ & 42,68 & 41,77 & 0,98 & $-0,91$ & 0,35 & $-0,11$ & $4-5$ \\
\hline & $09 \mathrm{H}$ & 42,68 & 41,77 & 0,98 & $-0,91$ & 0,35 & $-0,11$ & 4 \\
\hline & 09S & 42,68 & 41,77 & 0,98 & $-0,91$ & 0,35 & $-0,11$ & 4 \\
\hline \multirow{5}{*}{ Killuyuyo } & $11 \mathrm{~L}$ & 76,52 & 74,94 & 2,34 & $-1,58$ & 0,84 & $-1,5$ & $3-4$ \\
\hline & $11 \mathrm{~A}$ & 76,52 & 75,52 & 5,25 & -1 & $-0,71$ & 5,11 & $2-3$ \\
\hline & $11 \mathrm{~N}$ & 76,52 & 74,49 & 2,05 & $-2,03$ & 0,14 & $-0,25$ & $3-4$ \\
\hline & $11 \mathrm{H}$ & 76,52 & 74,49 & 2,05 & $-2,03$ & 0,14 & $-0,25$ & $4-5$ \\
\hline & $11 \mathrm{~S}$ & 76,52 & 74,49 & 2,05 & $-2,03$ & 0,14 & $-0,25$ & $4-5$ \\
\hline
\end{tabular}

*Número antes de la letra corresponde al código numérico de la muestra. La=Luminosidad antes de la prueba. Ld=Luminosidad después de la prueba. $\mathrm{L}=$ Prueba con respecto a la luz artificial. $\mathrm{A}=$ Prueba de lavado manual con jabón alcalino $(\mathrm{pH}=8) . \mathrm{N}=\mathrm{Prueba}$ de lavado manual con jabón neutro $(\mathrm{pH}=7) . \mathrm{H}=$ Prueba de frote en húmedo. $\mathrm{S}=$ Prueba de frote en seco. $\Delta \mathrm{E}=$ Diferencia total de color. $\Delta \mathrm{L} *=\mathrm{Va}-$ riación de la luminosidad. $\Delta \mathrm{a}^{*}=$ Variación en el rango rojo y verde. $\Delta \mathrm{b}^{*}=$ Variación en el rango amarillo y azul. 
Tabla 2. Lecturas del colorímetro antes y después de las pruebas aplicadas a los tejidos de lana de oveja y valoración según la escala de grises Table 2. Colorimeter readings before and after the tests applied to the sheep wool fabrics and the evaluation according to the gray scale.

\begin{tabular}{|c|c|c|c|c|c|c|c|c|}
\hline Lana & Codificación* & $\mathbf{L a}$ & Ld & $\Delta \mathbf{E}$ & $\Delta \mathbf{L}^{*}$ & $\Delta \mathbf{a}^{*}$ & $\Delta \mathbf{b}^{*}$ & Escala grises \\
\hline \multirow{5}{*}{ cochinilla rosa } & $01 \mathrm{~L}$ & 42,23 & 42,2 & 1,1 & $-0,33$ & $-0,88$ & 0,68 & 5 \\
\hline & $01 \mathrm{~A}$ & 41,43 & 33,58 & 10,54 & $-7,86$ & 6,47 & 2,75 & $1-2$ \\
\hline & $01 \mathrm{~N}$ & 42,23 & 42,96 & 0,88 & 0,74 & $-0,03$ & 0,48 & $4-5$ \\
\hline & $01 \mathrm{H}$ & 42,23 & 42,96 & 0,88 & 0,74 & $-0,03$ & 0,48 & $3-4$ \\
\hline & $01 \mathrm{~S}$ & 42,23 & 42,96 & 0,88 & 0,74 & $-0,03$ & 0,48 & $3-4$ \\
\hline \multirow{5}{*}{$\begin{array}{l}\text { cochinilla } \\
\text { naranja }\end{array}$} & 04L & 43,21 & 43 & 0,6 & $-0,21$ & $-0,43$ & $-0,35$ & $4-5$ \\
\hline & $04 \mathrm{~A}$ & 43,21 & 32,24 & 25,77 & $-10,97$ & $-4,75$ & $-22,83$ & 1 \\
\hline & $04 \mathrm{~N}$ & 43,21 & 43,13 & 1,48 & $-0,08$ & $-0,97$ & 1,1 & 4 \\
\hline & $04 \mathrm{H}$ & 43,21 & 43,13 & 1,48 & $-0,08$ & $-0,97$ & 1,1 & 4 \\
\hline & $04 \mathrm{~S}$ & 43,21 & 43,13 & 1,48 & $-0,08$ & $-0,97$ & 1,1 & 4 \\
\hline \multirow{5}{*}{ nogal } & 06L & 44,93 & 45,44 & 0,72 & 0,51 & 0,05 & $-0,51$ & $4-5$ \\
\hline & $06 \mathrm{~A}$ & 44,93 & 41,47 & 3,74 & $-3,46$ & 0,47 & $-1,35$ & 3 \\
\hline & $06 \mathrm{~N}$ & 44,93 & 42,62 & 2,45 & $-2,31$ & 0,8 & 0,06 & $3-4$ \\
\hline & $06 \mathrm{H}$ & 44,93 & 42,62 & 2,45 & $-2,31$ & 0,8 & 0,06 & $4-5$ \\
\hline & $06 \mathrm{~S}$ & 44,93 & 42,62 & 2,45 & $-2,31$ & 0,8 & 0,06 & $4-5$ \\
\hline \multirow{5}{*}{ Garau } & $08 \mathrm{~L}$ & 48,33 & 47,42 & 1,05 & $-0,91$ & 0,51 & 0,06 & $4-5$ \\
\hline & $08 \mathrm{~A}$ & 48,33 & 43,71 & 9,95 & $-4,62$ & 0,66 & 8,78 & $1-2$ \\
\hline & $08 \mathrm{~N}$ & 48,33 & 46,33 & 3,06 & -2 & 0,3 & 2,29 & $3-4$ \\
\hline & $08 \mathrm{H}$ & 48,33 & 46,33 & 3,06 & -2 & 0,3 & 2,29 & 4 \\
\hline & $08 \mathrm{~S}$ & 48,33 & 46,33 & 3,06 & -2 & 0,3 & 2,29 & 4 \\
\hline \multirow{5}{*}{ chilca } & $10 \mathrm{~L}$ & 28,65 & 27,98 & 0,76 & $-0,67$ & 0,19 & $-0,3$ & $4-5$ \\
\hline & $10 \mathrm{~A}$ & 28,65 & 23,86 & 9,26 & $-4,8$ & 6,88 & 3,92 & $1-2$ \\
\hline & $10 \mathrm{~N}$ & 28,65 & 27,5 & 1,55 & $-1,15$ & 1 & 0,27 & 4 \\
\hline & $10 \mathrm{H}$ & 28,65 & 27,5 & 1,55 & $-1,15$ & 1 & 0,27 & 4 \\
\hline & $10 \mathrm{~S}$ & 28,65 & 27,5 & 1,55 & $-1,15$ & 1 & 0,27 & 4 \\
\hline \multirow{5}{*}{ Killuyuyo } & $12 \mathrm{~L}$ & 68,41 & 68,33 & 0,52 & $-0,09$ & 0,03 & $-0,51$ & $4-5$ \\
\hline & $12 \mathrm{~A}$ & 68,41 & 62,7 & 6,26 & $-5,72$ & 2,23 & 1,23 & 2 \\
\hline & $12 \mathrm{~N}$ & 68,41 & 68,53 & 0,75 & 0,12 & 0,73 & $-0,17$ & $4-5$ \\
\hline & $12 \mathrm{H}$ & 68,41 & 68,53 & 0,75 & 0,12 & 0,73 & $-0,17$ & $4-5$ \\
\hline & $12 \mathrm{~S}$ & 68,41 & 68,53 & 0,75 & 0,12 & 0,73 & $-0,17$ & $4-5$ \\
\hline
\end{tabular}

* Número antes de la letra corresponde al código numérico de la muestra. La=Luminosidad antes de la prueba. Ld=Luminosidad después de la prueba. $\mathrm{L}=$ Prueba con respecto a la luz artificial. $\mathrm{A}=$ Prueba de lavado manual con jabón alcalino $(\mathrm{pH}=8)$. $\mathrm{N}=\mathrm{Prueba}$ de lavado manual con jabón neutro $(\mathrm{pH}=7)$. $\mathrm{H}=$ Prueba de frote en húmedo. $\mathrm{S}=$ Prueba de frote en seco. $\Delta \mathrm{E}=\mathrm{Diferencia}$ total de color. $\Delta \mathrm{L} *=$ Variación de la luminosidad. $\Delta \mathrm{a}^{*}=$ Variación en el rango rojo y verde. $\Delta \mathrm{b}^{*}=$ Variación en el rango amarillo y azul.

Los valores de la diferencia total de color de cada una de las probetas elaboradas con lana de oveja y fibra de alpaca se pueden analizar en la Figura 1. La mayor diferencia de color se presentó en la totalidad de las muestras cuando se aplicó el tratamiento de lavado con jabón alcalino $(\mathrm{pH}=8)$ y las muestras tinturadas con cochinilla son las que presentaron la mayor diferencia de color en relación a las demás muestras, esto puede deberse a que la cochinilla utilizada fue de tipo alimentario que es una cochinilla que se obtiene ya preparada y se la puede conseguir de los distribuidores del Perú para la industria alimentaria, esto de- bido a que en el país no se produce la cochinilla con fines comerciales y en algunas épocas del año no se la puede conseguir. Esta cochinilla según la descripción del fabricante presentaba una solubilidad del $50 \%$ en agua, y a esto puede deberse que no hubo una fijación optima en la fibra y se quedó en la superficie de la misma, produciéndose un tinturado irregular y eliminándose el colorante durante la prueba de lavado, ya que cuando la cochinilla es obtenida directamente del insecto desecado y molido presenta mejores resultados en cuanto a la solidez del color (Palacios-Ochoa y Ullauri, 2020; Vigueras y Portillo, 2001). 
La inestabilidad del color de todas las muestras, frente a la prueba de lavado con jabón alcalino puede deberse a que los tintes que se usaron son sensibles al cambio de $\mathrm{pH}$ del lado alcalino ya que en múltiples estudios se reporta la influencia del pH en el cambio de estructura química de los colorantes (Restrepo Baena et al., 2008) y por ende en el color (Garzón, 2008). El cambio de $\mathrm{pH}$ en la cochinilla que es la que presentó mayor diferencia de color fue de $\mathrm{pH} 5,5$ en el baño a un $\mathrm{pH}$ de 8 durante la prueba de lavado. La prueba que afectó menos a la estabilidad del color en los tejidos fue la de la exposición a la luz artificial, un resultado similar obtuvo Arroyo-Figueroa et al. (2017) al experimentar con el tinte de cebolla que resultó ser un tinte con mayor resistencia a la luz que a los otros factores que probaron, esto se puede explicar por las características de los componentes químicos de los tintes que se usaron en esta experimentación y que probablemente no son sensibles a la luz.

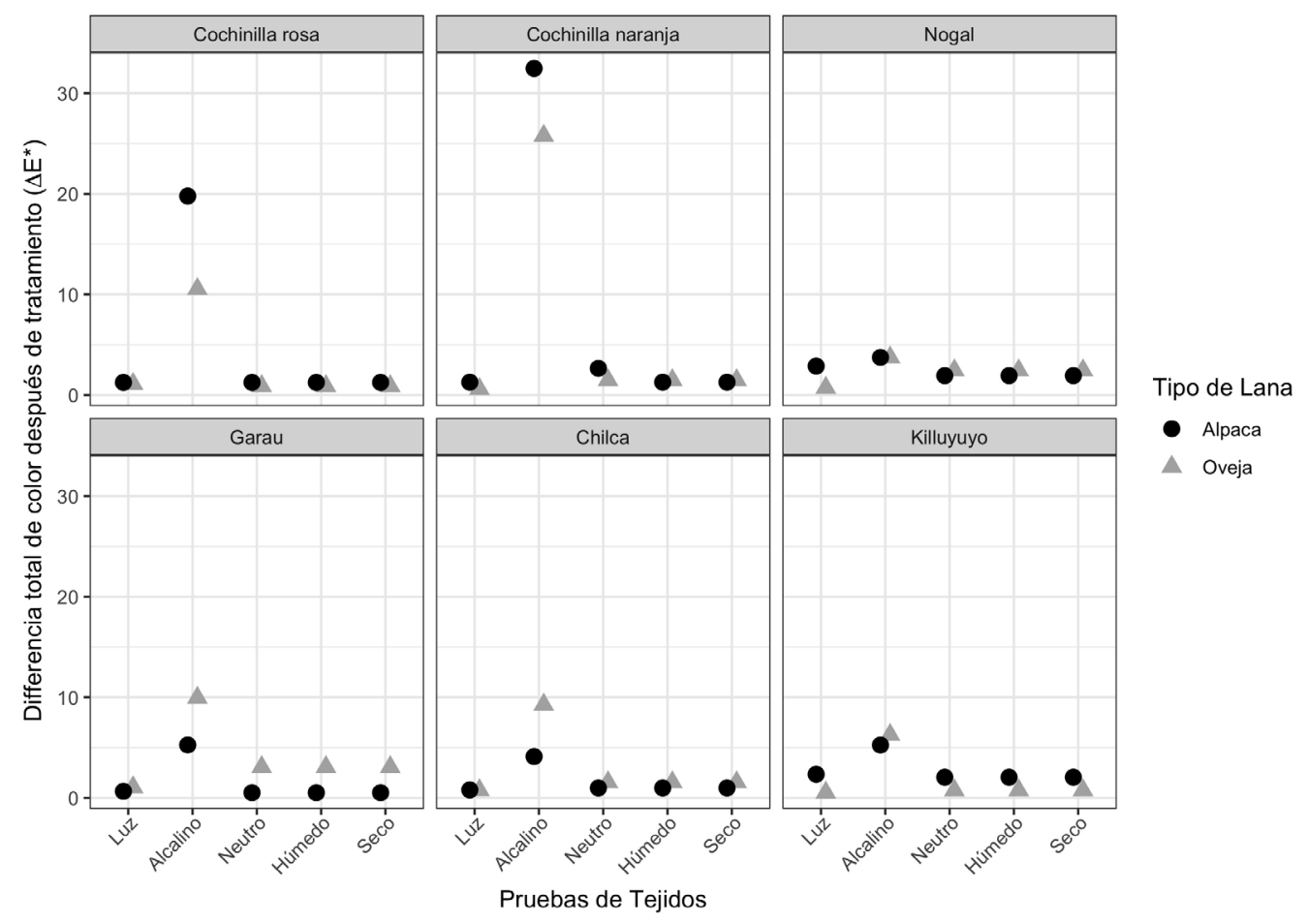

Figura 1. Diferencia total de color después de la aplicación de las pruebas de solidez a la luz (luz), lavado en jabón neutro (neutro) y alcalino (alcalino), frote en húmedo (húmedo) y seco (seco) en los tejidos con lana de oveja y fibra de alpaca cuyo color antes de aplicar el tinte fue el color natural

Figure 1. Total color difference after the application of light fastness (light), washing in neutral (neutral) and alkaline (alkaline) soap, wet (wet) and dry (dry) scrub tests on fabrics with sheep wool and alpaca fiber whose color before applying the dye was the natural color.

También se pudo demostrar mediante los valores de los componentes del color L, a y b de la escala CIEL*a*b*. que tonos más débiles son los obtenidos en los tejidos con fibra de alpaca y con todos los colorantes utilizados esto es con los extractos de nogal killuyuyo, garau, chilca y con la cochinilla, ya que los valores de L (luminosidad) son mayores en las muestras de tejido con hilo de alpaca, así en la muestra 01 que corresponde al tinturado con cochinilla en tono rosa el valor es de 53,26 y en la 02 de lana de oveja es de 42,23 y esa tendencia se observa en las demás muestras. Además, los tejidos de alpaca presentaron una mayor pérdida de color en todos los tratamientos, esto puede deberse a la estructura de la fibra, la misma que posee una medulación irregular lo que provoca una refracción de los colorantes y una menor fijación de los mismos a la fibra (Alonso, 2015).

Así mismo, se demostró que según las diferencias de los valores de los componentes de color a b y L de las diferentes muestras, como se puede apreciar en la Figura 2, la mayoría de muestras presentaron una pequeña diferencia de color, pero las teñidas con cochinilla tanto en color rosa como naranja son las que presentaron el mayor cambio en los componentes de color, en el rosa el cambio mayor se dio en el componente a y $\mathrm{L}\left(\Delta \mathrm{a}^{*}=\right.$ 6,47 y $\left.\Delta \mathrm{L}^{*}-7,86\right)$, tendiendo hacia el color rojo y más oscuro y en el naranja el mayor cambio fue en los componentes a, b y L del lado negativo $\left(\Delta \mathrm{a}^{*}-4,76, \Delta \mathrm{b}^{*}-22,83\right.$ y $\left.\Delta \mathrm{L}^{*}-10,97\right)$, es decir, el color cambió a uno con 
tendencia hacia el verde, azul y oscuro. Estos cambios en los componentes corresponden al tratamiento con jabón alcalino y a las muestras de fibra de alpaca, lo que se puede explicar también por las propiedades de la cochinilla utilizada, que produjo un teñido poco uniforme y lecturas de color en zonas más oscuras, además de la sensibilidad de la cochinilla al cambio de $\mathrm{pH}$ y la menor fijación debido a la estructura medulada de la fibra de alpaca (Alonso, 2015).

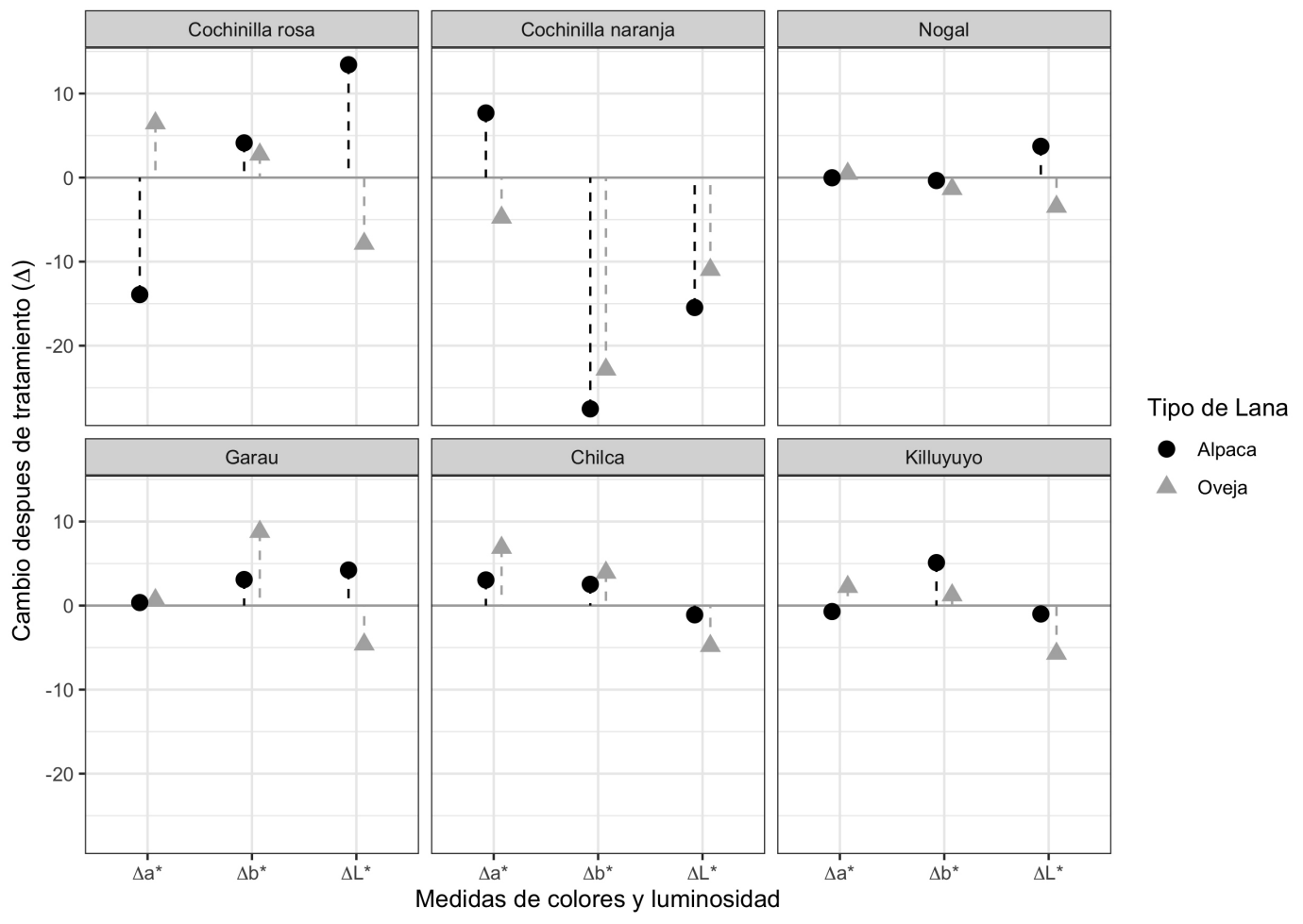

Figura 2. Diferencia en los valores de los componentes de color $\Delta \mathrm{a}^{*}, \Delta \mathrm{b}^{*} \mathrm{y} \Delta \mathrm{L}^{*}$ después de la aplicación de las pruebas de solidez en los tejidos de lana de oveja y fibra de alpaca.

Figure 2. Difference in the color component values $\Delta a^{*}, \Delta b^{*}$ and $\Delta L^{*}$ after the application of fastness tests on sheep wool and alpaca fiber fabrics.

Se puede afirmar que, en general, la pérdida de color estuvo dentro de rangos aceptables con la mayoría de los tratamientos aplicados, como se pudo determinar en la relación hecha con la escala de grises, ya que la casi totalidad de las muestras presentaron una valoración de 3 a 5 que es aceptable a excepción de las muestras teñidas con cochinilla que tuvieron una valoración de 1-2 en el tratamiento de lavado con jabón alcalino, según lo que se muestra en las Tablas 1 y 2 sobre los valores obtenidos utilizando la escala de grises.

\section{Conclusiones}

Las pruebas de solidez del color aplicadas a las probetas de tejidos de punto elaboradas con hilos de alpaca y lana de oveja, teñidos con los tintes extraídos del nogal, killuyuyo, garau, chilca y de la cochinilla, demostraron mayor estabilidad a la exposición a la luz artificial y una menor estabilidad a la prueba de lavado manual con jabón neutro y alcalino así como también a la prueba de frote en seco y en húmedo, observándose la mayor pérdida de color en el tratamiento de lavado con jabón alcalino. Estos datos son importantes porque sirven para incluirlos en las etiquetas de prendas que se elaboren con tejidos teñidos con el tipo de colorantes ensayados en este trabajo, los datos del etiquetado son de interés para la conservación de las prendas. Además, los ensayos sobre el tinturado sirven para incentivar a artesanos y diseñadores a la aplicación de los tintes naturales para la innovación de sus productos y puede darles la oportunidad de obtener productos únicos y con valor agregado. 


\section{Contribuciones de los autores}

- Rosa Cecilia Palacios Ochoa: conceptualización, adquisición de fondos, investigación, metodología, administración del proyecto, recursos, supervisión, validación, visualización, redacción-borrador original, redacción-revisión y edición.

- María Elisa Guillén Serrano: investigación.

- David Christopher Siddons: curación de datos, visualización.

\section{Referencias}

Alonso, J. (2015). Manual de control de calidad en productos textiles y afines. Universidad Politécnica de Madrid.

Arroyo-Figueroa, G., Álvarez-Canelo, J. G., Medina-Saavedra, T., Dzul-Cauih, J. (2017). Evaluación de la estabilidad del color en el teñido de lana y algodón con extracto de cebolla morada (Allium cepa). Revista Sistemas Experimentales, 4(19), 1-6. https://www.ecorfan.org/bolivia/researchjournals/Sistemas_Experimentales/vol4num10/Revista_de_Sistemas_Experimentales_V4_N10_1.pdf

Carrera-Gallissà, E. (2015). Caracterización de tejidos. Principales ensayos fisicos para evaluar la calidad de los tejidos textiles ( $1^{\mathrm{a}}$ ed.). Universitat Politècnica de Catalunya.

Garzón, G. A. (2008). Las antocianinas como colorantes naturales y compuestos bioactivos: Revisión. Acta Biológica Colombiana, 13(3), 27-36. https://revistas.unal.edu.co/index.php/actabiol/article/view/11337

Guirola, C. (2010). Tintes naturales y su uso en Mesoamérica desde la época prehispánica. Asociación Flaar Mesoamérica.

Jaramillo, H. (1988). Textiles y tintes. Centro Interamericano de Artesanías y Artes Populares.

Konica Minolta Sensing Americas, Inc. (2016). Entendiendo el espacio de color CIE L*A*B*. https://sensing. konicaminolta.us/mx/blog/entendiendo-el-espacio-de-color-cie-lab/\#: :text=El\%20espacio $\% 20 \mathrm{de} \% 20$ color\%20L,con\%20la\%20percepci\%C3\%B3n\%20visual\%20humana

Martínez, M. J. (2011). Aspectos técnicos de la fabricación de colorantes empleados en la vestimenta femenina de la época romana: fuentes escritas y experimentación. En C. Alfaro, M. J. Martínez García, \& J. Ortiz (eds.), Mujer y vestimenta. Aspectos de la identidad femenina en la antigüedad (pp. 185-212). Editorial Universidad de Valencia.

Norma ISO 105-C06:2010. Textiles. Ensayos de solidez del color. Parte C06: Solidez del color al lavado doméstico y comercial.

Palacios-Ochoa, C., \& Ullauri, N. (2020). Revalorización de métodos ancestrales de tinturado natural en las provincias de Loja y Azuay del sur de Ecuador. Siembra, 7(1), 050-059. https://doi.org/10.29166/siembra.v7il.1914

Restrepo Baena, O. J., Vázquez Jiménez, C. F., Bustamante Rúa, M. O. (2008). Influencia del pH en las propiedades pigmentarias de la goetita sintética. Dyna, 75(155), 163-170. https://revistas.unal.edu.co/index. $\mathrm{php} / \mathrm{dyna} /$ article/view/1751

Vigueras, A. L., Portillo, L. (2001). Usos del pigmento de la grana cochinilla. En C. Llanderal, \& R. Nieto (eds.), Cría de la grana cochinilla del nopal para la producción de pigmento (pp. 93-103). Editorial del Instituto de Fitosanidad. 